\title{
Virtual 3D Puzzles: A New Method for Exploring Geometric Models in VR
}

n many areas, learning involves understanding complex spatial phenomena. For example, engineers must master the construction of machines as a prerequisite for maintenance, and the spatial composition of molecules is an important task in chemistry. Medical students have considerable difficulties in imagining the spatial relations within the human body, which they must learn in anatomy. With interactive 3D computer graphics based on high-resolution geometric models, we can explore these spatial relations. To exploit this potential, we've combined some new dedicated 3D interaction and visualization techniques in a system inspired by a 3D puzzle.

\section{Why 3D puzzles?}

Traditionally, anatomy is taught using human models, anatomical atlases, or textbooks. Existing educational software systems like the Voxelman let students explore the visual human project's voxel data. Students can view the models from arbitrary positions and hide parts. However, these systems rely on visualization, and students are used to touching and directly interacting with objects. ${ }^{1}$ Some surgery training systems ${ }^{2}$ provide interactive manipulations, but they're mostly restricted to special tasks required for operative planning and intervention.

Our goal is different because we want to propose a method of actively investigating the models instead of performing virtual surgery. During our search for a convenient interaction method supporting this kind of exploration, we found that 3D puzzles provide a familiar concept for interacting with such complex models. In such a puzzle, users compose a set of elementary objects to form a specific model. The shape of these objects indicates which parts belong together. By intensively manipulating the objects, the user implicitly gains knowledge about the model's structure, which is exactly what we want to achieve.

\section{A virtual 3D puzzle}

By observing ourselves assembling a puzzle, we found that several issues are crucial to technically realizing a virtual 3D puzzle. ${ }^{3}$ To gain an overview of the problem, the user must be able to group and sort items on a set of trays. To make this possible, we provided a set of views where the user can place and group objects with dragand-drop operations (see Figure 1). Besides the main view where users compose the object, a detailed view
Felix Ritter and Thomas

Strothotte

Otto-von-

Guericke University of

Magdeburg,

Germany

Oliver Deussen

Dresden

University of

Technology,

Germany

Bernhard Preim

MeVis $\mathrm{gGmbH}$, Bremen, Germany

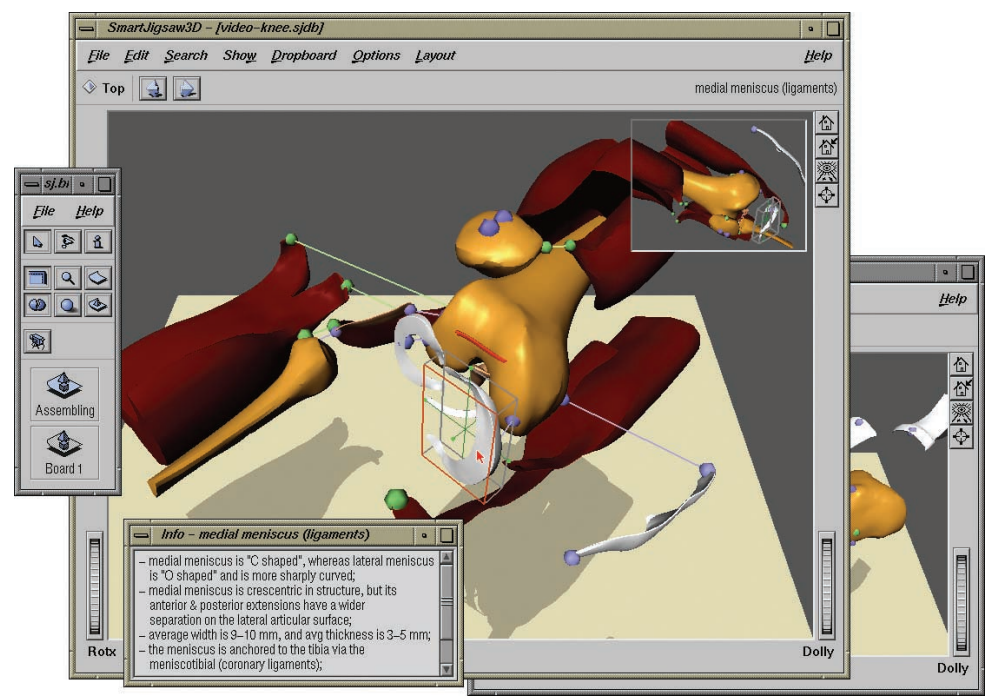

1 Overview of the system. The main view shows the so-far composed muscles, sinews, and bones. The unused items are placed randomly on another view, which is partly occluded on the right. The small panel on the left side lists all the 3D views. Additionally, the system offers textual information about the selected items (as seen at the bottom). 


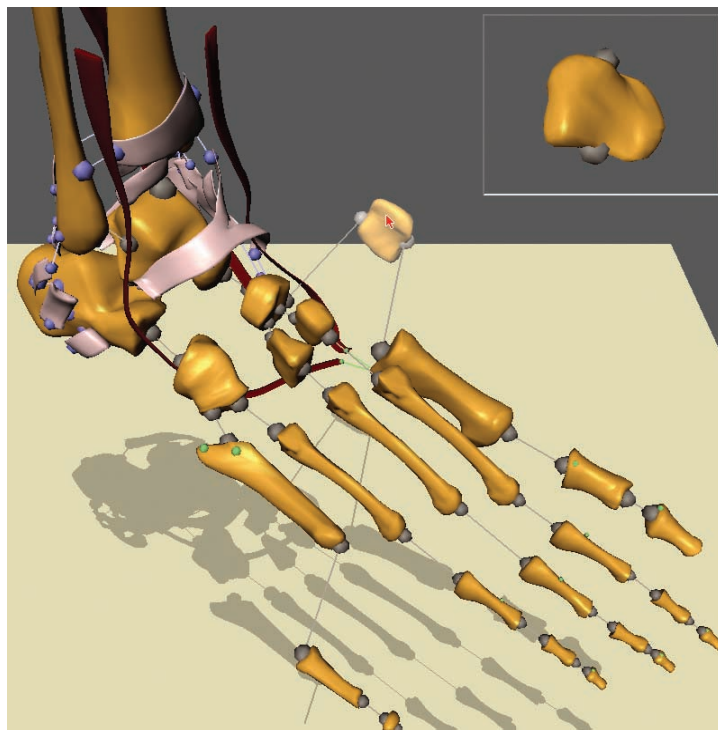

2 Exploded view of the partly composed model. We interconnected corresponding docking points within a certain range with lines. In the upper right, the system rotates the selected item to provide additional information about its shape.

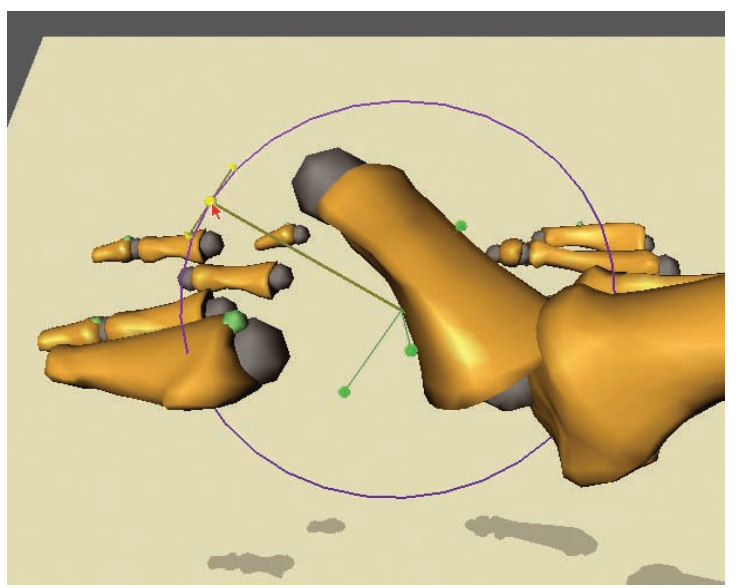

3 A user has snapped an object at one docking point. The transformation is now restricted to the rotation to correctly orient this object.

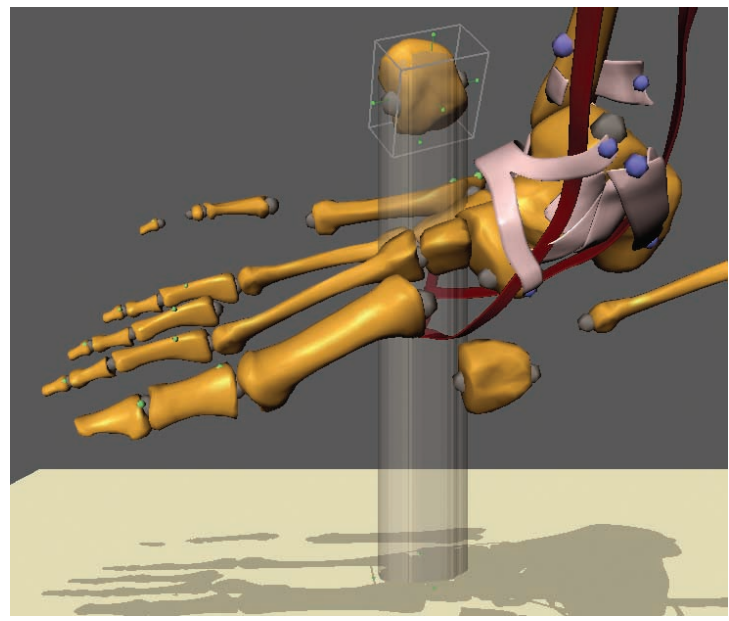

4 Semitransparent shadow volume supporting the spatial perception in relative positioning of objects. shows the currently selected object. The system rotates it automatically to help users see the shape (see upper right of Figure 2). A final view shows the composed model like the photos on the packages of real 3D puzzles, which help users find the right place for the puzzle pieces. Furthermore, an exploded view shows an exploded model so users can see the structure of the sofar-composed model (Figure 2).

\section{Transformation and connection}

Users must transform items precisely and connect them to solve a puzzle. A snapping mechanism and the users' bimanual skills (using both hands) support these tasks. The geometric model, which a puzzle author prepares beforehand, has a number of docking points that indicate connections between parts. Objects fit correctly if the docking points touch each other. The docking points' shape and color hint which objects connect.

Figure 3 shows the snapping mechanism. Objects snap together if their distance is less than a given threshold. If more than one docking point lies in the immediate vicinity, the behavior depends on the author's predetermination. If the system permits incorrect connections of objects, the object snaps to the closest docking point regardless of correctness. Once an object attaches, the same algorithm prevents the user from inadvertently detaching it. Separation is possible, however, with a quick movement.

The reverse snapping technique makes it difficult to attach an object to a wrong docking position. The opposite object impedes the composition by increasing the control-display ratio for movements toward an inappropriate docking point. Currently, the puzzle's author defines in the model's configuration whether to enable these mechanisms. This and other parameters let the author precisely determine the level of difficulty, ensuring the puzzle's solution isn't too easy or too hard.

People tend to use both hands when manipulating 3D objects. Medicine has also successfully applied twohanded interaction - for example, for preoperative neurosurgery planning. For interaction tasks, we can achieve the most intuitive handling with two-handed 3D interaction where the dominant hand (usually the right hand) does the fine-positioning relative to the nondominant hand. Our 3D puzzle supports simultaneous use of two input devices, a 3D mouse and a 2D mouse. Using these two input devices involves the users' bimanual motor skills, letting them perform dependent subtasks in compound tasks.

\section{Depth cues}

Because a 3D puzzle requires precise interaction in $3 \mathrm{D}$, simulating depth cues plays an important role. We used several techniques to provide the user with such cues. For example, we developed a shadow view with a light ground plane. We scaled this ground plane so that all objects cast a shadow on it, whereby the orientation remains fixed with regard to the camera.

A particularly useful technique for supporting the user during object translation is to connect the object and its casting shadow visually. The resultant shadow volume is rendered by semitransparent surfaces. 
This helps the users recognize the spatial relation between the object and its immediate neighborhood (see Figure 4).

Objects become highlighted when users touch them with the pointing device. The system displays the object name and category (such as muscles) in the upper part of the view. Double-clicking yields a short explanation of the object. The structure of these explanations is inspired by anatomical atlases, which provide this information to help readers understand the images.

Collision detection prevents users from moving objects through others. When objects collide, they're highlighted for a moment to provide visual feedback. If the user continues to attempt to move one object through another, the system initiates an acoustic signal and provides textual output in the status line. We incorporated the software library V-Collide for collision detection, which robustly accomplishes this.

\section{Experiences}

We discussed our 3D puzzle with recently qualified physicians and medical students, who have some computer experience but aren't used to learning systems and 3D interaction. After a short introduction to the system's goal and functionality, we asked the candidates to explore a geometric model of a foot and to compose muscles and sinews onto the skeleton.

All subjects liked the management of the different deposits and considered the snapping feature essential. Some would like the system to automatically compose a subset of the model in an animation, so they can watch and later repeat the task themselves.

Furthermore, the evaluation revealed that the composite 3D widget used for translating and rotating objects (Figure 3) doesn't intuitively convey its use. Three subjects had difficulties initializing rotation. For inexperienced users, an explicit representation might be more appropriate.

\section{Other applications}

Originally, we wanted the 3D puzzle to help users explore and compose geometric models. However, we can also use the puzzle to decompose a model. This can support rehearsing surgical procedures and preparation tasks in which users remove objects to expose a particular part.

Car mechanics in training must master the specific setup of complex engines. As an example, we prepared a six cylinder engine model and discussed the scenario with mechanical engineers (see Figure 5). The 3D puzzle can also help chemistry students and researchers understand spatial relation. Researchers might even be able to compose puzzle pieces, such as proteins, in a specific way to form new drugs.

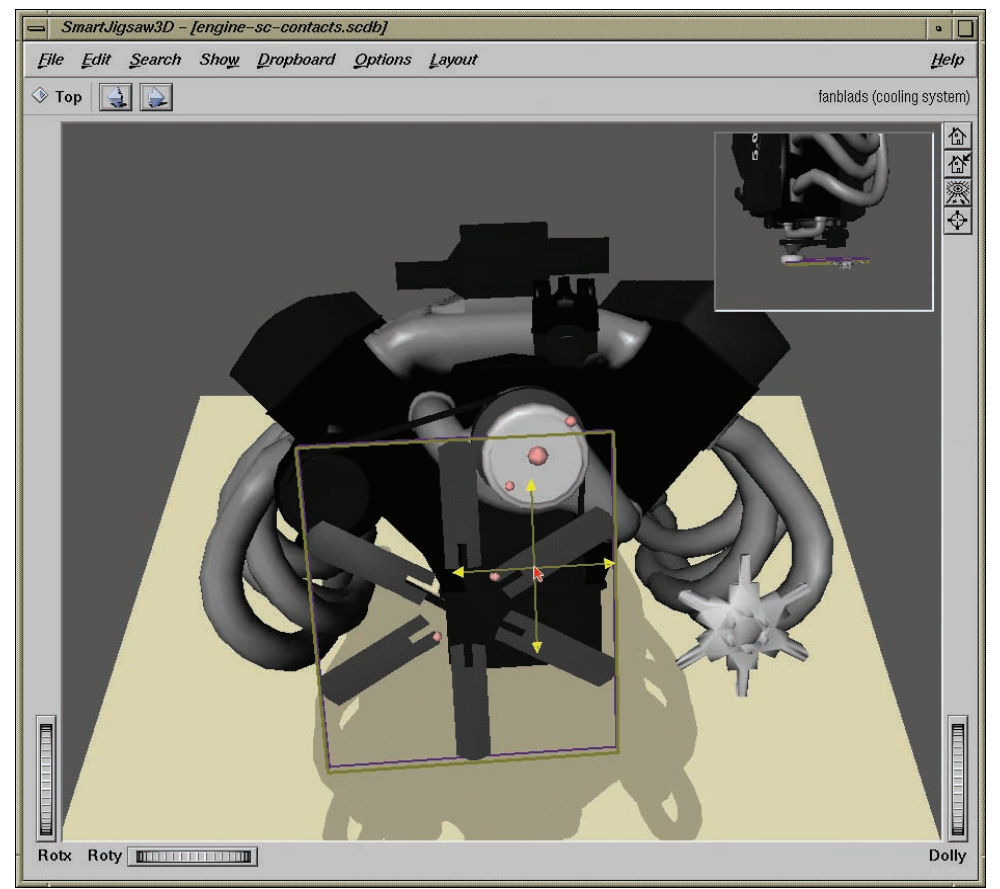

5 Complex car engine. The fan blades are being connected to the fan clutch. All docking points of the cooling system are spheres and have a uniform color.

In the future, we'll further investigate the power of our 3D puzzles. More content has to be provided to enable a broader user study. The metaphor will also serve as a platform for exploring new interaction methods for 3D models.

\section{References}

1. B. Preim, A. Raab, and T. Strothotte, "Coherent Zooming of Illustrations with 3D-Graphics and Textual Labels," Proc. Graphics Interface 1997, Morgan Kaufmann, San Francisco, 1997, pp. 105-113.

2. A. Sourin, O. Sourina, and H.T. Sen, "Virtual Orthopedic Surgery Training," IEEE Computer Graphics and Applications, vol. 20, no. 3, May/June 2000, pp. 6-9.

3. F. Ritter et al., "Using a 3D Puzzle as a Metaphor for Learning Spatial Relations," Proc. Graphics Interface 2000, Morgan Kaufmann, San Francisco, 2000, pp. 171-178.

Contact Ritter at fritter@isg.cs.uni-magdeburg.de, http://isgwww.cs.uni-magdeburg.de/ fritter/ 3dpuzzle.html.

Readers may contact either of the department editors by email at rosenblum@ait.nrl.navy.mil or Michael_ Macedonia@stricom.army.mil. 